\title{
Disruption of Mitochondrial Quality Control Genes Promotes Caspase-Resistant Cell Survival
}

\section{Following Apoptotic Stimuli}

Yulia Kushnareva $^{1}$, Vivian Moraes ${ }^{1}$, Julian Suess ${ }^{2}$, Bjoern Peters ${ }^{1}$, Donald D. Newmeyer ${ }^{1}$ and Tomomi Kuwana $^{1 *}$

${ }^{1}$ La Jolla Institute for Immunology

9420 Athena Circle, La Jolla, CA 92037, USA

${ }^{2}$ University of Konstanz, 78464 Konstanz, Germany

*Correspondence: tomomi@1ji.org

Key words: mitochondrial quality control, mitochondrial dynamics, mitophagy, mitochondrial heterogeneity, apoptosis, mitochondrial outer membrane permeabilization, siRNA screen, oncogenesis 


\begin{abstract}
In cells undergoing cell-intrinsic apoptosis, mitochondrial outer membrane permeabilization (MOMP) typically marks an irreversible step in the cell death process. However, in some cases a subpopulation of the treated cells can exhibit a sublethal response, termed "minority MOMP". In this phenomenon, the affected cells survive, despite a low level of caspase activation and a subsequent limited activation of the endonuclease CAD (DFFB). Consequently, these cells can experience DNA damage, increasing the probability of oncogenesis. To discover genes affecting MOMP response in individual cells, we conducted an imaging-based phenotypic siRNA screen. We identified multiple candidate genes whose downregulation increased the heterogeneity of MOMP within single cells. Among these were genes related to mitochondrial dynamics and mitophagy, which participate in the mitochondrial quality control (MQC) system. To test the hypothesis that functional MQC is important for reducing the frequency of minority MOMP, we developed an assay to measure the clonogenic survival of caspase-engaged cells. We found that cells deficient in various MQC genes were indeed prone to aberrant post-MOMP survival. Our data highlight the important role of proteins involved in mitochondrial dynamics and mitophagy in preventing apoptotic dysregulation and oncogenesis.
\end{abstract}

\title{
INTRODUCTION
}

Apoptosis is a ubiquitous cellular self-elimination process that is critical for the homeostasis of various cell populations. Dysregulated apoptosis is integral to cancer progression and contributes to multiple diseases, including immune and neurodegenerative disorders. Many cancer therapies rely on the enhanced apoptotic death of tumor cells. Apoptosis frequently involves a "cell-intrinsic" pathway involving mitochondria. The central event in mitochondria-dependent apoptosis is mitochondrial outer membrane permeabilization (MOMP), which is induced by BAX and BAK, the key pro-apoptotic BCL-2 family 
proteins $[1,2]$. BAK is constitutively located on the mitochondrial outer membrane (MOM), whereas BAX is mostly soluble in the cytoplasm. When cells receive an apoptotic stress signal, molecules belonging to a subset of the BCL-2 family termed "BH3-only proteins" activate BAX and BAK. Consequently, BAX translocates to the MOM, and both BAX and BAK integrate into the outer membrane and trigger MOMP by inducing the formation of large membrane pores [3-7]. In concert with these BCL2 family members, additional MOM proteins facilitate BAX-induced pore formation [4]. Anti-apoptotic BCL-2 family members (including BCL-2, BCL-xL, and MCL-1) inhibit MOMP by sequestering the BH3-only proteins and by antagonizing the pore-forming activity of BAX and BAK.

The apoptotic pores in the MOM allow proteins normally residing in the mitochondrial intermembrane space to escape into the cytoplasm. Certain of these proteins are apoptogenic, including cytochrome c, SMAC/DIABLO and OMI/HTRA-1. These proteins induce APAF-1-dependent activation of Caspase-9 and the effector caspases (most prominently Caspase-3), which cleave certain protein substrates that actively promote cell destruction and engulfment [8-10]. Even when caspase activity is blocked, MOMP per se usually leads to cell death, as it compromises energy metabolism in the permeabilized mitochondria [11-13]. Although mitochondrial membrane potential and cellular ATP are maintained for some time after MOMP, cells treated with caspase inhibitors typically exhibit a gradual decline in mitochondrial respiration and a complete loss of clonogenic survival over the course of two or three days [12].

Recent reports describe a phenomenon, termed "minority MOMP", in which cells exposed to relatively weak cell stressors can evade MOMP-dependent death [14-16]. In these atypical cells, only a fraction of the cell's mitochondria undergoes MOMP. As a result of minority MOMP, small amounts of cytochrome c and SMAC are released into the cytosol, and downstream caspases become activated at sub-lethal levels. 
This low-level caspase activation leads to a correspondingly limited activation of the apoptotic CAD (DFFB) endonuclease, which enters the cell nucleus and produces a degree of DNA damage. The surviving cells exhibit genome instability and an increased propensity to become oncogenic [17].

The mechanisms controlling the uniformity of MOMP in cells, and hence the frequency of minority MOMP, are unclear. To discover genes that affect MOMP, we conducted a focused high-content siRNA screen that analyzed 1318 genes that had been annotated in databases as having some relationship to mitochondria. A novel aspect of our screen was the identification of genes whose silencing produced a heterogeneous MOMP phenotype in which some mitochondria within the cell were permeabilized, while others remained intact. Our screen yielded functionally diverse candidate genes, including a significant group of genes associated with mitophagy, autophagy, and mitochondrial dynamics, the processes constituting the system of mitochondrial quality control (MQC) [18, 19]. We hypothesized that an increased heterogeneity of MOMP observed in MQC-compromised cells could increase the frequency of minority MOMP [17]. If so, downregulation of MQC-related genes would tend to allow cells to survive stresses that produce a degree of caspase activation, as a result of limited MOMP. To test this hypothesis, we developed a single-cell assay to measure the long-term clonogenic cell survival of cells that had exhibited a measurable degree of caspase activation, in response to treatment with a BH3-mimetic drug. Our results confirmed that deficiencies in various MQC proteins enhanced the survival of caspase-engaged cells. We conclude that mitochondrial dynsmics and mitophagy play a critical role in limiting the frequency of minority MOMP. Thus, proper functioning of the MQC system can be important to prevent oncogenesis that results from an incomplete execution of the mitochondrial apoptosis program.

\section{MATERIALS AND METHODS}




\section{Cell lines}

HeLa cells stably expressing Venus-BAX and OMI-mCherry cells [20] were constructed by the laboratory of Douglas Green (Department of Immunology, St. Jude Children Hospital). U2OS cells with APAF-1 CRISPR knockout (KO) were obtained from Dr. Stephen Tait (Beatson Institute, UK)[21] and Mitofusin (MFN) 1 and 2 double KO (DKO) cells were obtained from Dr. David Chan; California Institute of Technology). Immortalized OPA1 KO and wild type (WT) MEFs were obtained from ATCC (Manassas, VA, USA; deposited by Dr. David Chan). DRP1 KO MEFs were provided by Dr. Stefan Strack (University of Iowa); HeLa cells lacking five mitophagy receptors (TAX1BP1, NDP52, OPTN, NBR1, p62; Penta KO) were provided by Dr. Richard Youle (NIH) [22]. Unless indicated otherwise, cells were maintained in Dulbecco modified Eagles's medium (DMEM; Life Technologies) containing 10\% fetal bovine serum (GeminiBio) and $100 \mathrm{units} / \mathrm{ml}$ penicillin/streptomycin at $37^{\circ} \mathrm{C}$ with $5 \% \mathrm{CO}_{2}$.

\section{siRNA screen: cell transfections and treatments}

For the primary screen, gene-specific siRNA pools targeting 1318 mitochondria-annotated genes (Table S1) were cherry-picked from a Dharmacon genome-wide siRNA library (siGenome). The list of genes was generated based on a "mitochondria/mitochondrial membranes" queries in the in NIH compiled databases (http://www.ncbi.nlm.nih.gov/gene ). Pools of 4 individual gene-specific siRNAs and two nontargeting siRNAs were arrayed in 384-well master plates. Each plate also contained Dharmacon siGenome TOX and siGlo Red oligonucleotides as transfection indicators. Additionally, an siRNA pool targeting BAX was used as a positive control for the inhibition of apoptosis and BAX puncta formation. For reverse transfection, $4.4 \mu \mathrm{l}$ siRNA picked from each $1 \mu \mathrm{M}$ stock siRNA solution in the master plate was mixed with 30.6 $\mu$ l pre-diluted Lipofectamine RNAiMAX transfection reagent (Life Technology). RNAiMAX reagent was diluted 47 times in Gibco Opti-MEM reduced serum medium. The transfection mixtures (35 
$\mu \mathrm{l} /$ well) were incubated for 20 minutes at room temperature in a 384-well mixing plate. During the incubation period, Venus-BAX/OMI-mCherry HeLa cells were harvested by trypsinization and resuspended in antibiotic-free DMEM with 10\% FBS at 30,000 cells per ml. After incubation, lipid-siRNA complexes were dispensed into triplicate tissue/culture Costar black-wall clear bottom 384-well plates (10 $\mu \mathrm{l} /$ well); siRNAs targeting the same gene were separated in the different plates. Plate handling, transfection reagent/siRNA mixing, and dispensing were performed with Hamilton Star Automated Liquid Handler contained within a Baker Bioprotect class II biosafety cabinet. Cells were added at $40 \mu \mathrm{l}(1200$ cells) per well on top of the transfection mixture; final concentration of siRNA was $25 \mathrm{nM}$ in the $50 \mu \mathrm{l}$ per well volume. The plates were centrifuged at low speed $(\sim 300 \mathrm{x} g)$ for $1 \mathrm{~min}$ and transferred to a humidified $\mathrm{CO}_{2}$ incubator $\left(37^{\circ} \mathrm{C}\right)$. To minimize plate edge effects, the wells in two rows and columns at the plate edges were not used for transfections and contained only medium with cells. After $\sim 48 \mathrm{~h}$, the medium was aspirated and replaced with fresh culture medium containing $400 \mu \mathrm{M}$ etoposide (SigmaAldrich) and $20 \mu \mathrm{M}$ of the caspase inhibitor Q-VD (Q-VD-OPH; SM Biochemicals LLC, Anaheim, CA). Control wells were left untreated (no etoposide). By the time of etoposide treatment, most cells transfected with TOX siRNA had already shown morphological changes consistent with cell death, indicating efficient transfection. Following $24 \mathrm{~h}$ of etoposide treatment, the medium was removed, and cells were fixed with $0.5 \%$ glutaraldehyde as described previously [23]. After two washes with phosphate-buffered saline (PBS), cells were stained with Hoechst-3342 (Molecular Probes) diluted 1000 times in PBS. Plates were then washed twice with PBS, filled with $50 \mu \mathrm{PBS}$ per well, sealed and stored at $4{ }^{\circ} \mathrm{C}$. For the secondary screen, 4 individual siGENOME siRNAs per gene were obtained separately from Dharmacon, to target 95 gene candidates chosen from the primary screen. Individual siRNAs were arrayed in replicate 384-well plates, and the experiments were conducted as described above. 


\section{High-throughput image acquisition and analysis}

Image collection and processing were done using the Molecular Devices MetaXpress High Content Image Acquisition platform. Images of the cells were acquired in an Image Xpress Micro (IXM) device at 20X magnification with the following filters: DAPI (5060B), for Hoechst nuclear staining; YFP (2427A), for Venus-BAX fluorescence; and TexasRed (4040B), for OMI-mCherry fluorescence. (Numbers indicate the Semrock part number for the filters). Images were collected from 16 sites clustered in the center of the well. In our assay development, the punctate patterns of Venus-BAX translocation and OMI-mCherry retention in mitochondria were analyzed using the granularity module in MetaXpress (version 5.1) software. Individual cells were identified based on nuclear segmentation (DAPI channel images). Z' factor for the BAX-positive or BAX-negative phenotype assay was calculated as 0.85 based on etoposide-treated and untreated conditions as positive and negative controls, respectively.

High-throughput analysis of primary and secondary screen data was done with a customized algorithm (created as a "journal macro" in MetaXpress) developed for automated phenotype quantification. We configured image analysis to count the percentage of cells that satisfied either of two different criteria: 1) a given cell contains Venus-BAX granules of diameters within the expected diameter range, with total intensity above a certain threshold, or 2) a given cell contains both supra-threshold Venus-BAX and OMImCherry granules (not necessarily colocalized) within the appropriate diameter range. The thresholds were adjusted for stringency, to limit false-positive scores from background fluorescence and debris. Plate-to-plate variability was minimal for BAX foci and acceptably low for cells double-positive for BAX and OMI granules.

\section{Confocal microscopy}


Confocal images of Venus-BAX/OMI-mCherry cells were acquired with a $60 \mathrm{X}$ oil immersion objective on an Olympus FluoView FV10i automated confocal laser scanning microscope (Olympus Scientific Solutions America Corp, Waltham, MA, USA).

\section{CRISPR/Cas9-mediated gene depletion}

CRISPR experiments were performed using modified synthetic single guide (sg) RNAs from Synthego. Target sequences for guide RNAs were selected with the Synthego CRISPR design tool. RNA oligonucleotides were reconstituted in TE buffer (10 mM Tris-HCl, $1 \mathrm{mM}$ EDTA, $\mathrm{pH}$ 8.0) according to recommendations from the manufacturer. Ribonucleoprotein (RNP) complexes were formed from sgRNA and recombinant Cas9 2NLS protein (New England Biolabs) mixed at a sgRNA to Cas9 ratio of 4.5:1. After a 20-30 min incubation at room temperature, assembled RNP were delivered into cells by electroporation using a ThermoNeon ${ }^{\mathrm{TM}}$ device and $10 \mu \mathrm{M}$ tips (ThermoFisher). For one sample $\left(\sim 2 \times 10^{5}\right.$ cells), $3 \mu$ of sgRNA (from $30 \mathrm{mM}$ stock solution) were mixed with $1.5 \mu$ l Cas9 protein (from $20 \mu \mathrm{M}$ stock solution). U2OS cells were electroporated at $1230 \mathrm{v} / 10 \mathrm{~ms} / 4$ (pulse voltage, width, pulse number) settings. Immediately after electroporation, cells were transferred to 12 -well plate with pre-warmed antibiotic-free DMEM media with 10\% FBS. After two-three days of incubation, a portion of the control (unedited) and CRISPR cells were harvested for genomic DNA isolation using a QIAGEN genomic DNA purification kit. The remaining cells were left in culture for further propagation and clonal selection. Genomic DNA concentrations were measured using a NanoDrop instrument (ThermoFisher). Primer design and PCR amplification of the edited region were done according to Synthego recommendations. Sanger sequencing of PCR amplicons was performed at Genewiz or EtonBio; the resulting DNA sequencing chromatograms were analyzed using the Inference of CRISPR Edits (ICE) algorithm (Synthego). ICE analysis of CRISPR-edited genomic regions typically demonstrated at least $70 \%$ editing 
efficiency (i.e. 70\% knockout cells in the pool); sgRNA sequences used are shown Fig. S2. For clone selection, CRISPR cells were expanded for 2-3 additional days, harvested and subjected to sorting into 96-well plates using BD FACSAria-3 or FACSAria-4 Fusion instruments; typically, 1-4 single cells were dispensed into one well. After clonal expansion, cells were analyzed for knockout efficiency as described above. For each gene of interest, two clones with verified knockout were combined for use in further experiments.

\section{Assay for clonogenic survival after caspase activation via MOMP (outlined in Fig. 2)}

Cells (CRISPR knockout U2OS cells and MEFs (knockout and matched WT) were plated out at 3x10 per well containing $1 \mathrm{ml}$ media in 6-well plates the day before the experiment, 2 wells per condition. The cells were then treated with ABT-199 or ABT-737 at 2.5, 5 or $10 \mu \mathrm{M}$ for $5 \mathrm{~h}$. In the last 30 min of incubation, a caspase reporter dye, CellEvent Caspase3/7 Green (Life Technology; R37111), was added at $30 \mu \mathrm{l}$ per well. Cells were harvested with Trypsin/EDTA and washed with medium and PBS. Finally, cells were resuspended in $300 \mu \mathrm{l}$ of sorting buffer consisting of $1 \%$ BSA in PBS containing $20 \mu \mathrm{M}$ of QVD, where Q-VD was included to stop the caspase reaction. We noticed that, when these caspase-activated cells were left at room temperature or $4^{\circ} \mathrm{C}$ for more than $30-40 \mathrm{~min}$, their ability to survive was compromised. Therefore, care was taken to sort the cells immediately after harvesting. To minimize artifacts from sample handling delay, we operated two cell sorters (FACSAria; BD) simultaneously for wild type and gene-defective cells. Also, the sorting chamber and the plate holder were kept at $37^{\circ} \mathrm{C}$ during the run to prevent temperature shock to the cells. Six hundred cells within the population gated for green fluorescence were sorted in duplicate into a 48-well plate containing $300 \mu 1$ of conditioned medium per well. The cells were grown for 8-12 days, during which an additional $500 \mu 1$ of conditioned medium was added at day 4-5. Colonies were then stained with $6 \%$ glutaraldehyde containing $0.5 \%$ crystal violet. Non- 
treated cells distributed in the non-green gate were sorted as above and used as a control representing $100 \%$ growth. Because individual cell colonies tended to merge over time, we measured the total area occupied by colonies, using the ImageJ macros developed by Guzman et al [24]. The percentage of surviving caspase-engaged cells in the total population was normalized against the $100 \%$ growth control. The data are summarized from 2 to 3 independent experiments, as indicated. P values were calculated using the mean and the standard deviation in each set of KO and WT cells with two-way ANOVA analysis using Prism 7 (Graphpad Software).

\section{RESULTS AND DISCUSSION}

\section{Screening strategy and assay development}

To identify novel cell-intrinsic regulators of MOMP, we designed a high-content image-based siRNA screen. As we were primarily interested in mitochondrial function, we carried out a focused siRNA screen targeting genes with annotated relationships to mitochondria (Table S1). Our strategy took advantage of a HeLa cell line expressing two fluorescent reporter proteins (described previously by Llambi et al [20]) that enabled us to simultaneously interrogate both BAX activation and MOMP. In living cells, VenusBAX is cytoplasmic (diffuse), and OMI-mCherry is localized to mitochondria. When apoptosis is induced, Venus-Bax is translocated to mitochondria, and OMI-mCherry is released into the cytoplasm and degraded. Normally, once apoptotic BAX translocation is initiated in a given cell, mitochondrial intermembrane space proteins (including cytochrome c or OMI) are released in a synchronous, rapid fashion $[14,20,25]$. The release of proteins from the mitochondrial interior can only be restricted under special circumstances, e.g. cristae junction remodeling by overexpression of a mutant form of the optic 
atrophy 1 (OPA1) protein [26] or MOMP inhibition by a recently described endolysosome-linked mechanism [27]. However, in some cells the release of intermembrane proteins is not an all-or-none event and mitochondria within the same cell display heterogeneity in MOMP response [16, 17]. We hypothesized that downregulation of certain genes could increase MOMP heterogeneity and promote postMOMP cell survival, even in caspase proficient cells.

To begin to test our hypothesis, we analyzed punctate mitochondrial distribution of Venus-BAX and OMImCherry in cells treated with the apoptosis inducer, etoposide. The caspase inhibitor Q-VD was included in these experiments to decrease premature cell loss and detachment. We configured our automated image analysis to quantify three cellular phenotypes: 1) apoptotic cells with mitochondria that have undergone MOMP - containing translocated BAX and lacking intramitochondrial OMI (BAX puncta-positive); 2) non-apoptotic cells with intact mitochondria lacking BAX and containing OMI (BAX puncta-negative), and 3) atypical cells with mitochondria containing BAX but which have not released OMI (BAX/OMI puncta double-positive cells) (Fig 1. A, B). Untreated (no etoposide) cells transfected with control siRNA were non-apoptotic and showed normal mitochondrial morphology (Fig. 1A, E) indicating that our optimized transfection conditions produced minimal toxicity. As expected, most etoposide-treated cells fell into either of the first two categories, while BAX/OMI double-positive cells were minimally present (Fig.1 A, E). In assay validation experiments, we tested the effect of OPA1 siRNA. OPA1 mediates mitochondrial fusion in conjunction with its cleavage by the mitochondrial protease OMA1 [28-30] and has multiple roles in apoptotic signaling [23, 26, 31-33]. Knockdown of OPA1 protein increased the number of BAX/OMI double-positive cells compared with cells transfected with a non-targeting siRNA (Fig. 1F). Furthermore, high-resolution confocal imaging of OMA1 siRNA-treated cells revealed mitochondrial heterogeneity in the MOMP response: in individual BAX/OMI double-positive cells, some 
mitochondria contained translocated BAX (green puncta) and lost OMI, whereas other mitochondria lacked BAX and retained OMI (red puncta) (Fig. 1D).

A BAX siRNA pool (which targets both endogenous BAX and ectopic Venus-BAX expression) potently inhibited etoposide-induced apoptosis and markedly reduced Venus-BAX fluorescence (Fig. 1C, F). However, knockdown of BAK did not inhibit apoptosis, indicating that in these etoposide-treated cells, MOMP was predominantly BAX-dependent. Transfection efficiency in high-throughput screening experiments was verified using a cell death-inducing transfection marker (siTOX) that consistently produced $\sim 90 \%$ loss of cells within 2 days post-transfection (not shown). Efficient knockdown of other proteins produced by siRNA pools was confirmed in supplementary experiments (Fig. S1A).

\section{The siRNA screen uncovers potential regulators of MOMP}

The image-based assay was used to screen 1318 gene-specific siRNA pools. We ranked gene scores in three groups corresponding to the cell phenotype categories noted above: genes whose knockdown I) increased the percentage of Venus-BAX puncta; II) decreased the percentage of cells with Venus-BAX puncta; or III) increase the percentage of BAX/OMI double-positive cells. When we applied a $10 \%$ cutoff in the score distribution range, we identified $\sim 200$ initial "hits" (with scores $\sim 3-5$ fold above or below controls for the Categories I and II). Hits in the Category III were from the top $10 \%$ tail of the score distribution (Fig. 1G). For a secondary screen, we chose to re-assay 95 of the primary screen hits, based on their effect scores and our judgment concerning their biological interest (we decided not to pursue some "housekeeping" genes.) In this secondary screen, we tested the four siRNAs from each siRNA pool individually. To reduce the likelihood of off-target effects, we required at least 3 of the 4 individual 
siRNAs to give concordant results [34]. Applying this more stringent criterion yielded a final list of 63 candidate genes (Table S3). Several hits in Categories I and II were consistent with previous reports (Table S2). For example, the effect of siRNA targeting BNIP3 (Category II) is consistent with the known cell death-promoting activity of this protein [35]. Acting in the opposite manner, the siRNAs scoring in Category I increased the percentage of cells undergoing MOMP. For example, several candidate genes in this group are required for metabolism of the mitochondrial lipid, cardiolipin (PLSCR3, PRELID1, and TRIAP1). Although cardiolipin is important for $\mathrm{BAX}$ pore formation $[6,36]$, in certain paradigms cardiolipin deficiency potentiates the release of apoptogenic proteins [37, 38]. In particular, the deficiency of p53-regulated protein TRIAP1 impaired cardiolipin level in mitochondria, compromised bioenergetics and potentiated cytochrome c release [38]. Potential novel regulators of MOMP include metabolic enzymes ACADL (long-chain acyl-coenzyme A dehydrogenase) which catalyzes one of the early steps in the circle of mitochondrial beta-oxidation of fatty acids, and FAHD1 (fumarylacetoacetate hydrolase domain-containing protein) with putative oxaloacetate decarboxylase activity in mitochondria. The roles of these proteins in mitochondrial metabolism and cell senescence are emerging [39, 40] and their inhibitory effects on MOMP could be of interest for further investigations. In this study, we did not further pursue the hits in the Categories I and II but focused on the unique phenotype produced by the hits in category III. Of note, in nearly all cells containing both BAX and OMI puncta, BAX and OMI were not colocalized, implying that some mitochondria within a cell had undergone MOMP, while others had not (similar to the phenotype shown in Fig. 1D). Perhaps the most striking outcome of our screen is that a majority of hits in the Category III are related to the mitochondrial quality control (MQC) system.

\section{The interplay of MQC and MOMP}


The concept of MQC is that mitochondrial dynamics (fission and fusion) work in tandem with mitophagy (the autophagic elimination of dysfunctional mitochondrial fragments) to maintain mitochondrial structural and functional integrity. If defective mitochondria cannot be repaired through fusion with functional organelles, they are prone to excessive fragmentation and elimination by mitophagy. It is postulated that asymmetrical fission segregates defective mitochondria, making the mitochondrial population inherently heterogeneous. These isolated small mitochondria are then redirected into a preautophagic pool [41, 42]. In cells dysfunctional for mitochondrial dynamics or mitophagy, damaged mitochondria would be predicted to accumulate, and the organelles would become abnormally heterogeneous with respect to bioenergetic function and the distribution of proteins involved in MOMP $[43,44]$. Our screen tended to confirm this prediction.

\section{Downregulation of mitophagy-related genes promotes MOMP heterogeneity}

Among the hits in Category III, both ubiquitin-dependent (e.g. ATG12, MUL1) and ubiquitin-independent (e.g. FUNDC1, BNIP3L, BLOC1S1/GCN5L1, TRIAP1, MARCH5) mitophagy mechanisms [45-48] were represented (Table 1). ATG12 is ubiquitin-like protein that is integral for general autophagy and has additional roles in apoptosis and cell survival [49]. For example, ATG12 deficiency compromises mitochondrial function and promotes cellular oncogenic transformation [50]. One of the top-scoring hits was BLOC1S1/GCN5L1, which is reported to have multiple functions, including the coordinate regulation of mitochondrial biogenesis and mitophagy, through protein acetylation [51]. BNIP3L/NIX (originally described as a BCL-2-related pro-apoptotic protein) and FUNDC1 directly interact with the LC3 protein in the autophagosome formation pathway and promote mitophagy facilitated by hypoxic conditions [48, $50,52,53]$ 


\section{Other MQC-related hits in the Category III}

Besides well-defined components of mitophagy pathways, our screen yielded multiple candidates that could affect MQC as part of their function. For example, PTPIP51 (encoded by the RMDN3 gene) was shown to be important for mitochondria-endoplasmic reticulum (ER) tethering, and therefore, its putative role in MQC and apoptosis could be linked to multiple functions regulated by ER-mitochondrial contacts, such as ER stress-induced $\mathrm{Ca}^{2+}$ release [54], autophagosome formation and mitochondrial fission [55, 56].

Another functional group represented by multiple hits in the screen (ATCAY, DISC1, TRAK1 and SPATA19) involves mitochondrial motility on microtubules, in which motor proteins such as Kinesin 1 interact with certain mitochondrial membrane proteins, e.g. MIRO1 [57, 58]. We suspect that these genes appeared as hits in our screen because microtubule-based motility is important for mitochondrial fusion, including the transient fusion event known as "kiss and run" [59,60]. Since fusion is a key element of MQC, a loss of mitochondrial motility would be expected to increase the functional heterogeneity of the mitochondrial population within each cell.

Other interesting candidates include multifunctional E3 ubiquitin ligases RNF5 and MARCH5. Mitochondrial targets and pathways regulated by RNF5 remain to be identified. MARCH5 reportedly regulates activities of mitofusin-1 (MFN1) and dynamin-related protein 1 (DRP1) or its outer membrane receptor MFF, the key mediators of mitochondrial fusion and fission, respectively [61-63]. However, DRP1 and MFN themselves did not show significant effects in our screen, perhaps due to the redundancy. Among multifunctional proteins that may influence mitochondrial dynamics indirectly, LETM1 is the mitochondrial $\mathrm{Ca}^{2+} / \mathrm{H}^{+}$antiporter with pleiotropic effects on ATP production, mitophagy, cristae structure and mitochondria morphology [64]. In particular, mitochondria lacking LETM1 are prone to undergo 
DRP1-independent fission [65]. In our screen, LETM1 knockdown also increased mitochondrial fragmentation (not shown) as well as MOMP heterogeneity. Other candidate genes in the Category III are highlighted in Table 1. Overall, our screen suggests that a deficiency in MQC disturbs the normal apoptotic response and, in particular, promotes heterogeneous MOMP.

\section{An assay to measure clonogenic cell survival despite caspase activation}

Based on the number of hits our screen being involved in MQC, we hypothesized that MQC-dependent mitochondrial heterogeneity could result in an increased frequency of “minority MOMP”. In this scenario, when a minor fraction of mitochondria undergoes MOMP, the resulting sub-lethal caspase activation leads to DNA damage and promotes oncogenesis [17]. To test our hypothesis, we developed a clonogenic survival assay based on that described by Ichim et al. [17] (Fig. 2). In this assay, we induced apoptosis with a BH3-mimetic compound, ABT-737 or ABT-199, incubated the cells with a fluorescent caspasereporter compound, and used FACS to sort out cells that exhibited caspase activation. We then plated the cells and, after several days in culture, quantified the percentage of cells that survived and formed colonies.

If the caspase dye faithfully reports MOMP-induced caspase activation, rather than unspecific staining and/or caspase activation independent of MOMP, we would predict that cells deficient in caspase activation via the intrinsic pathways would not display cell survival in this assay. To confirm this, we compared APAF1 CRISPR KO U2OS cells to their parental WT control cells. APAF1 is required to activate Caspase-9 and subsequently the effector Caspases-3 and -7, following the MOMP-dependent release of cytochrome c, SMAC and OMI. As shown in Fig 3, only a trace amount of cells were stained by the dye in the APAF1 KO cell population treated with a BH3 mimetic, ABT-737 (Fig. 3A). In contrast, the WT cells showed a population of cells stained with the dye. The same number of dye-stained cells in 
the gate was collected and subject to a colony formation assay. The percentage of the surviving cells in the entire population was then calculated. APAF1-deficient cells from this gate did not survive clonogenically (Fig. 3B), suggesting that the staining in these cells is not a result of genuine caspase activation. We conclude that our clonogenic assay reflects true MOMP-dependent survival of caspaseengaged cells and thus indicates the occurrence of minority MOMP.

\section{MQC deficiency promotes minority MOMP}

To test further whether MQC is important to mitigate minority MOMP, we used the clonogenic assay described above to investigate the effects of deleting selected candidate genes from our screen as well as other known MQC factors. We generated CRISPR cells (U2OS) with perturbed expression of ATG12, RMDN3 (PTPIP51) and BNIP3L. Knockdown of each of these genes produced MOMP heterogeneity in our imaging assay (Table 1). To obtain stable knockout cells, we generated CRISPR-edited cell pools and derived clonal populations from single cells (as described in Methods). Validation of the CRISPR-based gene editing efficiency is shown in Fig. S2. As shown in Fig. 4, we found that cells depleted of either RMDN3 or ATG12 showed significantly higher post-MOMP survival than WT cells. However, BNIP3L KO did not show an increase in minority MOMP. Reasons for this are unknown but could be related to other apoptosis-related activities of BNIP3L.

To examine further the hypothesis that compromised MQC promotes minority MOMP, we performed experiments using previously derived cells in which genes known to be directly involved in mitochondrial dynamics or mitophagy had been deleted, namely MFN1 and 2 double knockout (DKO), OPA1 KO, DRP1 KO MEFs and "penta-KO" HeLa cells that had been shown to be deficient in mitophagy due to the deletion 
of 5 mitophagy receptor genes [22]. As shown in Fig. 5, all of these MQC-deficient cells exhibited an enhancement of cell survival in our clonogenic assay, compared with their matched controls. (The effect in DRP1 KO cells was not statistically significant; in this case, a weaker effect could reflect the slowing of cell proliferation that was reported with DRP1 deletion [66]. Thus, the loss of these genes important for MQC promoted the proliferative survival of caspase-engaged cells, again implying an increased frequency of minority MOMP.

\section{Conclusion}

Taken together, our data allow us to conclude that the MQC system is critical for mitigating the phenomenon of minority MOMP. This result has implications for oncogenesis: even a small increase in cell viability resulting from sublethal caspase activation could potentially raise the frequency of oncogenic cell transformation $[67,68]$. Therefore, treatments developed to limit minority MOMP could improve the cytotoxic effects of anti-cancer treatments. For example, proteins that are known to inhibit mitophagy, such as SIAH3 [22], are potential therapeutic targets. Also, we predict, based on our observation that placing sorted caspase-engaged cells on ice eliminated their ability to survive clonogenically (see Methods), that cryotherapy could prevent minority MOMP when used in combination with pro-apoptotic cancer therapeutics such as BH3 mimetic drugs. In conclusion, our study provides new insights into the mechanisms of aberrant apoptosis that are important for developing therapeutic strategies.

\section{ACKNOWLEDGMENTS}

We thank the Flow Cytometry Core Facility at La Jolla Institute for their expert help in developing the clonogenic assay. We thank Drs. Stephen Tait (Cancer Research UK Beatson Institute, University of 


\section{Glasgow, UK), Sonia Sharma (Functional Genomics Center at LJI) and Alexander Andreyev (The Scripps Research Institute) for helpful discussions and critical reading of the manuscript. This work was supported by R21CA216304 and a SPARK award from the LJI Board of Directors to D.D.N.}

\begin{tabular}{|c|c|c|}
\hline Gene & score & Function \\
\hline RNF5 & 85.3 & $\begin{array}{l}\text { E3 ubiquitin ligase; ER quality control; translocates from ER to mitochondrial in antiviral response; controls UPR, autophagy, anti-tumor immunity } \\
{[69-72]}\end{array}$ \\
\hline LETM1 & 78.8 & $\begin{array}{l}\text { Mitochondrial } \mathrm{Ca}^{2+} \text { and/or } \mathrm{K}^{+} \text {transport; implicated in assembly of ETC supercomplexes and maintenance of tubular mitochondrial morphology } \\
{[64,73,74]}\end{array}$ \\
\hline MARCH5 & 64 & $\begin{array}{l}\text { E3 ubiquitin ligase, regulates mitophagy and mitochondrial dynamics via targeting FUNDC1, MFN1/2 and Fis1; involved in MAVS signaling [75- } \\
\text { 77] }\end{array}$ \\
\hline FUNDC1 & 59.3 & $\begin{array}{l}\text { Regulation of LC3-dependent autophagy and parkin-independent mitophagy; a MARCH } 5 \text { target; regulates MQC in hypoxia, interacts with Drp1 } \\
{[46,75,78]}\end{array}$ \\
\hline DISC1 & 50.3 & $\begin{array}{l}\text { Disrupted-in-schizophrenia-1; enriched in MAM; controls Mitofilin stability, regulates mitochondrial trafficking, } \mathrm{Ca}^{2+} \text { dynamics, and bioenergetics } \\
\text { [79-81] }\end{array}$ \\
\hline ULK1 & 48.3 & $\begin{array}{l}\text { Autophagy activating kinase, phosphorylates FUNDC1; interacts with Bcl-2-L13 and LC3B to induce mitophagy; negatively regulated by mTOR } \\
\text { [82-84] }\end{array}$ \\
\hline ATG12 & 42.8 & $\begin{array}{l}\text { Integral component of general and selective autophagy, binding partner of ATG5; promotes apoptosis; regulates mitochondrial biogenesis [14, } 85 \text {, } \\
86]\end{array}$ \\
\hline RMDN3 & 39.5 & $\begin{array}{l}\text { Encodes tyrosine phosphatase-interacting protein-51 (PTPIP51); controls mitochondria-ER tethering, ER stress, autophagy, parkin-dependent } \\
\text { mitophagy [54-56] }\end{array}$ \\
\hline $\mathrm{NR} 2 \mathrm{C} 2$ & 38.7 & $\begin{array}{l}\text { Nuclear receptor/transcriptional regulator (also known as TR4); DNA repair function; KO promotes tumorigenesis, complex I deficiency, } \\
\text { myophathy [87-89] }\end{array}$ \\
\hline IER3 & 37 & $\begin{array}{l}\text { Early response gene, multifunctional; positive and negative apoptosis regulator, tumor-suppressive activity; interacts with Mcl-1; modulates } \\
\text { complex V [90-92] }\end{array}$ \\
\hline SLC25A14 & 33.3 & $\begin{array}{l}\text { Encodes UCP5; mitochondrial metabolite/anion transporter; KD decreases } \Delta \Psi \text {; implicated in ROS production, and DJ-1-dependent } \\
\text { neuroprotection[93-95] }\end{array}$ \\
\hline PHB & 33.3 & Prohibitin, controls ETC protein stability, OMA1/OPA1-dependent fusion and cristae morphogenesis, apoptosis, cancer cell proliferation [96-98] \\
\hline MRPL12 & 33 & $\begin{array}{l}\text { Mitochondrial ribosomal protein, regulates mitochondrial gene expression; upregulated in cancer; mutations are linked to a mitochondrial disease } \\
\text { [99-101] }\end{array}$ \\
\hline BNIP3L & 30 & $\begin{array}{l}\text { Atypical pro-apoptotic Bcl-2 family protein, multifunctional, mediates hypoxia-activated autophagy and mitophagy with effects on mitochondrial } \\
\text { fission }[48,52,102]\end{array}$ \\
\hline GPER1 & 24 & G-protein coupled estrogen receptor \\
\hline TMEM127 & 24 & $\begin{array}{l}\text { Endosome-associated tumor suppressor gene, linked to various cancers; regulates Rab5-mediated endosomal fusion [103]; mitochondrial function is } \\
\text { unknown }\end{array}$ \\
\hline ATCAY & 23.7 & Encodes caytaxin, a brain-specific kinesin interacting protein; involved in kinesin-dependent mitochondrial motility on microtubules [104] \\
\hline TRIAP1 & 22 & $\begin{array}{l}\text { p53-regulated antiapoptotic factor, linked to tumorigenesis; KD promotes MOMP and fission; lipid transfer activity, cardiolipin biosynthesis [38, } \\
105,106]\end{array}$ \\
\hline C19orf12 & 16.3 & $\begin{array}{l}\text { an orphan mitochondrial protein whose loss of function is linked to neurodegenerative diseases; involved in lipid metabolism, stimulates autophagy } \\
{[107,108]}\end{array}$ \\
\hline SPATA19 & 15 & Spermatogenesis associated 19; important for sperm motility by regulating mitochondrial structure and function [109] \\
\hline BLOC1S1 & 9.5 & $\begin{array}{l}\text { General control of amino acid synthesis } 5 \text { like-1 (GCN5L1/BLOC1S1); endosome-lysosomal function; mitochondrial protein acetylation and } \\
\text { mitophagy [51] }\end{array}$ \\
\hline TRAC1 & 7 & Essential for kinesin 1-depencent mitochondrial trafficking $[57,58]$ \\
\hline
\end{tabular}


Table 1. A list of genes whose downregulation increased mitochondrial heterogeneity (BAX/OMIpositive phenotype) in siRNA screen. MQC functions (mitochondrial dynamics, mitophagy) of the hits are highlighted in red. Other highlighted functions that can affect mitochondrial heterogeneity include regulation of mitochondrial respiration and apoptosis. Genes in boldface (RMDN3, ATG12, and BNIP3L) were selected for further experiments. Numbers indicate assay scores obtained in the secondary screen. Other secondary screen hits are listed in Table S2.

\section{REFERENCES}

1. Chipuk, J.E., et al., The BCL-2 family reunion. Mol Cell, 2010. 37(3): p. 299-310.

2. Youle, R.J. and A. Strasser, The BCL-2 protein family: opposing activities that mediate cell death. Nat Rev Mol Cell Biol, 2008. 9(1): p. 47-59.

3. Gillies, L.A., et al., Visual and functional demonstration of growing Bax-induced pores in mitochondrial outer membranes. Mol Biol Cell, 2015. 26(2): p. 339-49.

4. Kushnareva, Y., et al., Bax Activation Initiates the Assembly of a Multimeric Catalyst that Facilitates Bax Pore Formation in Mitochondrial Outer Membranes. PLoS Biol, 2012. 10(9): p. e1001394.

5. Kuwana, T., et al., Pro-apoptotic Bax molecules densely populate the edges of membrane pores. Sci Rep, 2016. 6: p. 27299.

6. Kuwana, T., et al., Bid, Bax, and lipids cooperate to form supramolecular openings in the outer mitochondrial membrane. Cell, 2002. 111(3): p. 331-42.

7. Ader, N.R., et al., Molecular and topological reorganizations in mitochondrial architecture interplay during Bax-mediated steps of apoptosis. Elife, 2019. 8.

8. Nicholson, D.W. and N.A. Thornberry, Caspases: killer proteases. Trends Biochem Sci, 1997. 22(8): p. 299-306.

9. Bratton, S.B. and G.S. Salvesen, Regulation of the Apaf-1-caspase-9 apoptosome. J Cell Sci, 2010. 123(Pt 19): p. 3209-14.

10. Ricci, J.E., et al., Disruption of mitochondrial function during apoptosis is mediated by caspase cleavage of the $p 75$ subunit of complex I of the electron transport chain. Cell, 2004. 117(6): $p$. 773-86.

11. Kushnareva, Y. and D.D. Newmeyer, Bioenergetics and cell death. Annals of the New York Academy of Sciences, 2010. 1201: p. 50-7.

12. Lartigue, L., et al., Caspase-independent mitochondrial cell death results from loss of respiration, not cytotoxic protein release. Mol Biol Cell, 2009. 20(23): p. 4871-84.

13. Colell, A., et al., GAPDH and autophagy preserve survival after apoptotic cytochrome c release in the absence of caspase activation. Cell, 2007. 129(5): p. 983-97.

14. Tait, S.W. and D.R. Green, Mitochondrial regulation of cell death. Cold Spring Harb Perspect Biol, 2013. 5(9). 
15. Gong, Y.N., et al., To the edge of cell death and back. FEBS J, 2019. 286(3): p. 430-440.

16. Tait, S.W., et al., Resistance to caspase-independent cell death requires persistence of intact mitochondria. Dev Cell, 2010. 18(5): p. 802-13.

17. Ichim, G., et al., Limited mitochondrial permeabilization causes DNA damage and genomic instability in the absence of cell death. Mol Cell, 2015. 57(5): p. 860-872.

18. Ni, H.M., J.A. Williams, and W.X. Ding, Mitochondrial dynamics and mitochondrial quality control. Redox Biol, 2015. 4: p. 6-13.

19. Pickles, S., P. Vigie, and R.J. Youle, Mitophagy and Quality Control Mechanisms in Mitochondrial Maintenance. Curr Biol, 2018. 28(4): p. R170-R185.

20. Llambi, F., et al., $A$ unified model of mammalian BCL-2 protein family interactions at the mitochondria. Mol Cell, 2011. 44(4): p. 517-31.

21. Ichim, G., et al., Limited mitochondrial permeabilization causes DNA damage and genomic instability in the absence of cell death. Mol Cell, 2015. 57(5): p. 860-72.

22. Lazarou, M., et al., The ubiquitin kinase PINK1 recruits autophagy receptors to induce mitophagy. Nature, 2015. 524(7565): p. 309-314.

23. Kushnareva, Y., et al., Mitochondrial dysfunction in an Opa1(Q285STOP) mouse model of dominant optic atrophy results from Opa1 haploinsufficiency. Cell Death Dis, 2016. 7: p. e2309.

24. Guzman, C., et al., ColonyArea: an ImageJ plugin to automatically quantify colony formation in clonogenic assays. PLoS One, 2014. 9(3): p. e92444.

25. Goldstein, J.C., et al., The coordinate release of cytochrome c during apoptosis is rapid, complete and kinetically invariant. Nat Cell Biol, 2000. 2(3): p. 156-62.

26. Yamaguchi, R., et al., Opa1-mediated cristae opening is Bax/Bak and BH3 dependent, required for apoptosis, and independent of Bak oligomerization. Mol Cell, 2008. 31(4): p. 557-69.

27. Wang, T.S., et al., Endolysosomal Targeting of Mitochondria Is Integral to BAX-Mediated Mitochondrial Permeabilization during Apoptosis Signaling. Dev Cell, 2020. 53(6): p. 627-645 e7.

28. Ehses, S., et al., Regulation of OPA1 processing and mitochondrial fusion by $m$-AAA protease isoenzymes and OMA1. J Cell Biol, 2009. 187(7): p. 1023-36.

29. Head, B., et al., Inducible proteolytic inactivation of OPA1 mediated by the OMA1 protease in mammalian cells. J Cell Biol, 2009. 187(7): p. 959-66.

30. Jiang, X., et al., Activation of mitochondrial protease OMA1 by Bax and Bak promotes cytochrome c release during apoptosis. Proc Natl Acad Sci U S A, 2014. 111(41): p. 14782-7.

31. Frezza, C., et al., OPA1 controls apoptotic cristae remodeling independently from mitochondrial fusion. Cell, 2006. 126(1): p. 177-89.

32. Scorrano, L., et al., A distinct pathway remodels mitochondrial cristae and mobilizes cytochrome c during apoptosis. Dev Cell, 2002. 2(1): p. 55-67.

33. Kushnareva, Y.E., et al., Loss of OPA1 disturbs cellular calcium homeostasis and sensitizes for excitotoxicity. Cell Death Differ, 2013. 20(2): p. 353-65.

34. Sigoillot, F.D. and R.W. King, Vigilance and validation: Keys to success in RNAi screening. ACS Chem Biol, 2011. 6(1): p. 47-60.

35. Chinnadurai, G., S. Vijayalingam, and S.B. Gibson, BNIP3 subfamily BH3-only proteins: mitochondrial stress sensors in normal and pathological functions. Oncogene, 2008. 27 Suppl 1: p. S114-27. 
36. Landeta, O., et al., Reconstitution of proapoptotic BAK function in liposomes reveals a dual role for mitochondrial lipids in the BAK-driven membrane permeabilization process. J Biol Chem, 2011. 286(10): p. 8213-8230.

37. Choi, S.Y., et al., Cardiolipin deficiency releases cytochrome c from the inner mitochondrial membrane and accelerates stimuli-elicited apoptosis. Cell Death Differ, 2007. 14(3): p. 597-606.

38. Potting, C., et al., TRIAP1/PRELI complexes prevent apoptosis by mediating intramitochondrial transport of phosphatidic acid. Cell Metab, 2013. 18(2): p. 287-95.

39. Etemad, S., et al., Oxaloacetate decarboxylase FAHD1 - a new regulator of mitochondrial function and senescence. Mech Ageing Dev, 2019. 177: p. 22-29.

40. Softic, S., et al., Dietary Sugars Alter Hepatic Fatty Acid Oxidation via Transcriptional and Posttranslational Modifications of Mitochondrial Proteins. Cell Metab, 2019. 30(4): p. 735-753 e4.

41. Twig, G., et al., Fission and selective fusion govern mitochondrial segregation and elimination by autophagy. EMBO J, 2008. 27(2): p. 433-46.

42. Shirihai, O.S., M. Song, and G.W. Dorn, 2nd, How mitochondrial dynamism orchestrates mitophagy. Circ Res, 2015. 116(11): p. 1835-49.

43. Chen, H., A. Chomyn, and D.C. Chan, Disruption of fusion results in mitochondrial heterogeneity and dysfunction. J Biol Chem, 2005. 280(28): p. 26185-92.

44. Weaver, D., et al., Distribution and apoptotic function of outer membrane proteins depend on mitochondrial fusion. Mol Cell, 2014. 54(5): p. 870-8.

45. Narendra, D., et al., Parkin is recruited selectively to impaired mitochondria and promotes their autophagy. J Cell Biol, 2008. 183(5): p. 795-803.

46. Liu, L., et al., Mitochondrial outer-membrane protein FUNDC1 mediates hypoxia-induced mitophagy in mammalian cells. Nat Cell Biol, 2012. 14(2): p. 177-85.

47. Levine, B. and J. Yuan, Autophagy in cell death: an innocent convict? J Clin Invest, 2005. 115(10): p. 2679-88.

48. Hamacher-Brady, A. and N.R. Brady, Mitophagy programs: mechanisms and physiological implications of mitochondrial targeting by autophagy. Cell Mol Life Sci, 2016. 73(4): p. 775-95.

49. Subramani, S. and V. Malhotra, Non-autophagic roles of autophagy-related proteins. EMBO Rep, 2013. 14(2): p. 143-51.

50. Liu, H., et al., ATG12 deficiency leads to tumor cell oncosis owing to diminished mitochondrial biogenesis and reduced cellular bioenergetics. Cell Death Differ, 2019.

51. Scott, I., et al., GCN5L1/BLOS1 Links Acetylation, Organelle Remodeling, and Metabolism. Trends Cell Biol, 2018. 28(5): p. 346-355.

52. Youle, R.J. and D.P. Narendra, Mechanisms of mitophagy. Nat Rev Mol Cell Biol, 2011. 12(1): p. 9-14.

53. Hanna, R.A., et al., Microtubule-associated protein 1 light chain 3 (LC3) interacts with Bnip3 protein to selectively remove endoplasmic reticulum and mitochondria via autophagy. J Biol Chem, 2012. 287(23): p. 19094-104.

54. Stoica, R., et al., ER-mitochondria associations are regulated by the VAPB-PTPIP51 interaction and are disrupted by ALS/FTD-associated TDP-43. Nat Commun, 2014. 5: p. 3996.

55. Gomez-Suaga, P., S. Paillusson, and C.C.J. Miller, ER-mitochondria signaling regulates autophagy. Autophagy, 2017. 13(7): p. 1250-1251.

56. Puri, R., et al., Mul1 restrains Parkin-mediated mitophagy in mature neurons by maintaining ERmitochondrial contacts. Nat Commun, 2019. 10(1): p. 3645. 
57. Henrichs, V., et al., Mitochondria-adaptor TRAK1 promotes kinesin-1 driven transport in crowded environments. Nat Commun, 2020. 11(1): p. 3123.

58. Zhao, Y., et al., Metaxins are core components of mitochondrial transport adaptor complexes. Nat Commun, 2021. 12(1): p. 83.

59. Lee, C.A., L.S. Chin, and L. Li, Hypertonia-linked protein Trak1 functions with mitofusins to promote mitochondrial tethering and fusion. Protein Cell, 2018. 9(8): p. 693-716.

60. Liu, X., et al., Mitochondrial 'kiss-and-run': interplay between mitochondrial motility and fusionfission dynamics. Embo J, 2009. 28(20): p. 3074-89.

61. Park, Y.Y., et al., Loss of MARCH5 mitochondrial E3 ubiquitin ligase induces cellular senescence through dynamin-related protein 1 and mitofusin 1. J Cell Sci, 2010. 123(Pt 4): p. 619-26.

62. Nakamura, N., et al., MARCH-V is a novel mitofusin 2- and Drp1-binding protein able to change mitochondrial morphology. EMBO Rep, 2006. 7(10): p. 1019-22.

63. Cherok, E., et al., Novel regulatory roles of Mff and Drp1 in E3 ubiquitin ligase MARCH5dependent degradation of MiD49 and Mcl1 and control of mitochondrial dynamics. Mol Biol Cell, 2017. 28(3): p. 396-410.

64. Li, Y., et al., LETM1 is required for mitochondrial homeostasis and cellular viability (Review). Mol Med Rep, 2019. 19(5): p. 3367-3375.

65. Dimmer, K.S., et al., LETM1, deleted in Wolf-Hirschhorn syndrome is required for normal mitochondrial morphology and cellular viability. Hum Mol Genet, 2008. 17(2): p. 201-14.

66. Wakabayashi, J., et al., The dynamin-related GTPase Drp1 is required for embryonic and brain development in mice. J Cell Biol, 2009. 186(6): p. 805-16.

67. Ichim, G. and S.W. Tait, A fate worse than death: apoptosis as an oncogenic process. Nat Rev Cancer, 2016. 16(8): p. 539-48.

68. Liu, X., et al., Caspase-3 promotes genetic instability and carcinogenesis. Mol Cell, 2015. 58(2): p. 284-96.

69. Kuang, E., J. Qi, and Z. Ronai, Emerging roles of E3 ubiquitin ligases in autophagy. Trends Biochem Sci, 2013. 38(9): p. 453-60.

70. Jeon, Y.J., et al., Regulation of glutamine carrier proteins by RNF5 determines breast cancer response to ER stress-inducing chemotherapies. Cancer Cell, 2015. 27(3): p. 354-69.

71. Zhong, B., et al., The ubiquitin ligase RNF5 regulates antiviral responses by mediating degradation of the adaptor protein MITA. Immunity, 2009. 30(3): p. 397-407.

72. $\mathrm{Li}, \mathrm{Y}$., et al., Gut microbiota dependent anti-tumor immunity restricts melanoma growth in Rnf5(-/-) mice. Nat Commun, 2019. 10(1): p. 1492.

73. Jiang, D., L. Zhao, and D.E. Clapham, Genome-wide RNAi screen identifies Letm1 as a mitochondrial Ca2+/H+ antiporter. Science, 2009. 326(5949): p. 144-7.

74. Tamai, S., et al., Characterization of the mitochondrial protein LETM1, which maintains the mitochondrial tubular shapes and interacts with the AAA-ATPase BCS1L. J Cell Sci, 2008. 121(Pt 15): p. 2588-600.

75. Chen, Z., et al., Mitochondrial E3 ligase MARCH5 regulates FUNDC1 to fine-tune hypoxic mitophagy. EMBO Rep, 2017. 18(3): p. 495-509.

76. Yoo, Y.S., et al., The mitochondrial ubiquitin ligase MARCH5 resolves MAVS aggregates during antiviral signalling. Nat Commun, 2015. 6: p. 7910.

77. Tanaka, A., et al., Proteasome and p97 mediate mitophagy and degradation of mitofusins induced by Parkin. J Cell Biol, 2010. 191(7): p. 1367-80. 
78. Wu, W., et al., FUNDC1 regulates mitochondrial dynamics at the ER-mitochondrial contact site under hypoxic conditions. EMBO J, 2016. 35(13): p. 1368-84.

79. Park, Y.U., et al., Disrupted-in-schizophrenia 1 (DISC1) plays essential roles in mitochondria in collaboration with Mitofilin. Proc Natl Acad Sci U S A, 2010. 107(41): p. 17785-90.

80. Park, S.J., et al., DISC1 Modulates Neuronal Stress Responses by Gate-Keeping ER-Mitochondria $\mathrm{Ca}(2+)$ Transfer through the MAM. Cell Rep, 2017. 21(10): p. 2748-2759.

81. Norkett, R., F. Lesept, and J.T. Kittler, DISC1 Regulates Mitochondrial Trafficking in a Miro1-GTPDependent Manner. Front Cell Dev Biol, 2020. 8: p. 449.

82. Murakawa, T., et al., A Mammalian Mitophagy Receptor, Bcl2-L-13, Recruits the ULK1 Complex to Induce Mitophagy. Cell Rep, 2019. 26(2): p. 338-345 e6.

83. Kundu, M., ULK1, mammalian target of rapamycin, and mitochondria: linking nutrient availability and autophagy. Antioxid Redox Signal, 2011. 14(10): p. 1953-8.

84. Wu, W., et al., ULK1 translocates to mitochondria and phosphorylates FUNDC1 to regulate mitophagy. EMBO Rep, 2014. 15(5): p. 566-75.

85. Liu, H., et al., ATG12 deficiency leads to tumor cell oncosis owing to diminished mitochondrial biogenesis and reduced cellular bioenergetics. Cell Death Differ, 2020. 27(6): p. 1965-1980.

86. Rubinstein, A.D., et al., The autophagy protein Atg12 associates with antiapoptotic Bcl-2 family members to promote mitochondrial apoptosis. Mol Cell, 2011. 44(5): p. 698-709.

87. Liu, S., et al., Mice lacking TR4 nuclear receptor develop mitochondrial myopathy with deficiency in complex I. Mol Endocrinol, 2011. 25(8): p. 1301-10.

88. Shen, J., et al., TR4 nuclear receptor enhances the cisplatin chemo-sensitivity via altering the ATF3 expression to better suppress HCC cell growth. Oncotarget, 2016. 7(22): p. 32088-99.

89. Lin, S.J., et al., Minireview: Pathophysiological roles of the TR4 nuclear receptor: lessons learned from mice lacking TR4. Mol Endocrinol, 2014. 28(6): p. 805-21.

90. Arlt, A. and $\mathrm{H}$. Schafer, Role of the immediate early response 3 (IER3) gene in cellular stress response, inflammation and tumorigenesis. Eur J Cell Biol, 2011. 90(6-7): p. 545-52.

91. Jin, $\mathrm{H}$., et al., IER3 is a crucial mediator of TAp73beta-induced apoptosis in cervical cancer and confers etoposide sensitivity. Sci Rep, 2015. 5: p. 8367.

92. Stachel, I., et al., Modulation of nuclear factor E2-related factor-2 (Nrf2) activation by the stress response gene immediate early response-3 (IER3) in colonic epithelial cells: a novel mechanism of cellular adaption to inflammatory stress. J Biol Chem, 2014. 289(4): p. 1917-29.

93. Guzman, J.N., et al., Oxidant stress evoked by pacemaking in dopaminergic neurons is attenuated by DJ-1. Nature, 2010. 468(7324): p. 696-700.

94. Gorgoglione, R., et al., The human uncoupling proteins 5 and 6 (UCP5/SLC25A14 and UCP6/SLC25A30) transport sulfur oxyanions, phosphate and dicarboxylates. Biochim Biophys Acta Bioenerg, 2019. 1860(9): p. 724-733.

95. Senapedis, W.T., et al., Whole genome siRNA cell-based screen links mitochondria to Akt signaling network through uncoupling of electron transport chain. Mol Biol Cell, 2011. 22(10): p. 1791-805.

96. Osman, C., C. Merkwirth, and T. Langer, Prohibitins and the functional compartmentalization of mitochondrial membranes. J Cell Sci, 2009. 122(Pt 21): p. 3823-30.

97. Sievers, C., et al., Prohibitins are required for cancer cell proliferation and adhesion. PLoS One, 2010. 5(9): p. e12735. 
98. Anderson, C.J., et al., Prohibitin levels regulate OMA1 activity and turnover in neurons. Cell Death Differ, 2020. 27(6): p. 1896-1906.

99. Wang, Z., J. Cotney, and G.S. Shadel, Human mitochondrial ribosomal protein MRPL12 interacts directly with mitochondrial RNA polymerase to modulate mitochondrial gene expression. J Biol Chem, 2007. 282(17): p. 12610-8.

100. Serre, V., et al., Mutations in mitochondrial ribosomal protein MRPL12 leads to growth retardation, neurological deterioration and mitochondrial translation deficiency. Biochim Biophys Acta, 2013. 1832(8): p. 1304-12.

101. Zhang, Q., et al., Differentially expressed mitochondrial genes in breast cancer cells: Potential new targets for anti-cancer therapies. Gene, 2017. 596: p. 45-52.

102. Chiche, J., et al., Hypoxic enlarged mitochondria protect cancer cells from apoptotic stimuli. J Cell Physiol, 2010. 222(3): p. 648-57.

103. Qin, Y., et al., The tumor susceptibility gene TMEM127 is mutated in renal cell carcinomas and modulates endolysosomal function. Hum Mol Genet, 2014. 23(9): p. 2428-39.

104. Aoyama, T., et al., Cayman ataxia protein caytaxin is transported by kinesin along neurites through binding to kinesin light chains. J Cell Sci, 2009. 122(Pt 22): p. 4177-85.

105. Li, Y., et al., Overexpression of Mitochondria Mediator Gene TRIAP1 by miR-320b Loss Is Associated with Progression in Nasopharyngeal Carcinoma. PLoS Genet, 2016. 12(7): p. e1006183.

106. Andrysik, Z., et al., A genetic screen identifies TCF3/E2A and TRIAP1 as pathway-specific regulators of the cellular response to $p 53$ activation. Cell Rep, 2013. 3(5): p. 1346-54.

107. Venco, P., et al., Mutations of C19orf12, coding for a transmembrane glycine zipper containing mitochondrial protein, cause mis-localization of the protein, inability to respond to oxidative stress and increased mitochondrial Ca(2)(+). Front Genet, 2015. 6: p. 185.

108. Hartig, M.B., et al., Absence of an orphan mitochondrial protein, c19orf12, causes a distinct clinical subtype of neurodegeneration with brain iron accumulation. Am J Hum Genet, 2011. 89(4): p. 543-50.

109. Mi, Y., Z. Shi, and J. Li, Spata19 is critical for sperm mitochondrial function and male fertility. Mol Reprod Dev, 2015. 82(11): p. 907-13.

\section{FIGURE LEGENDS}

Figure 1. Imaging-based screening assay for regulators of apoptotic MOMP response. (A) Representative epifluorescence microscopy of untreated and etoposide-treated cells expressing VenusBAX (green; YFP channel) and OMI-mCherry (red; Texas Red channel). Bottom panel illustrates enlarged cells with indicated phenotypes. Nuclei were stained with Hoechst 33342 (blue; DAPI channel). 
(B) Identification of Venus-BAX and OMI-mCherry puncta using MetaXpress granularity application module. A representative enlarged image of etoposide-treated cells (left panel) and corresponding image segmentations. The granularity module identifies Venus-BAX (middle panel) and OMI-mCherry puncta. Note that cells with BAX "granules" do not contain OMI "granules", and vice versa. Nuclear segmentation settings correctly identify fragmented (apoptotic) and normal size nuclei. (C) BAX siRNA inhibits VenusBAX puncta formation and OMI-mCherry release in etoposide-treated cells. (D) A confocal image of live Venus-BAX/OMI-mCherry cells transfected OMA1 siRNA. Arrows indicate cells with heterogeneous MOMP. (E, F) Examples of phenotype quantification using granularity application module and highthroughput microscopy. (E) Quantification of indicated phenotypes in untreated and etoposide-treated cells transfected with a non-targeting (control) siRNA; "non-determined" phenotype corresponds to a small fraction of non-fluorescent cells. (F). Effects of indicated siRNAs on the phenotype distribution. Data are mean \pm S.E.M. ( $n=3$ replicate wells). (G) Sorted assay scores for the primary screen siRNA set in triplicate plates. Numbers indicate the percentage of cells positive for both Venus-BAX and OMImCherry foci. Values shown are mean and SEM, $n=3$. Hits were identified from the top $10 \%$ tail of the BAX/OMI score.

\section{Figure 2. Cell-based 'minority MOMP' assay}

Cells were treated with ABT199 or ABT737 for $5 \mathrm{~h}$ and a caspase dye was added for the final 30 min.

Cells were harvested and 600 cells from the FITC-positive gate were sorted into 48-well plates containing the conditioned media. Grown colonies were stained with crystal violet and colony areas were measured using the macro developed by Guzman et al. [24](see Methods)

Figure 3. Survived cells had APAF1-dependent caspase activation 
(A) Dot-plots of wild-type U2OS and APAF1 CRISPR KO cells stained with a caspase dye (CellEvent; Life Technologies) after treatment with ABT737 at 2.5, 5 or $10 \mu \mathrm{M}$ for $5 \mathrm{~h}$. (B) Calculated percentages of surviving cells in WT U2OS cells and APAF1 KO are shown. Data presented are from two independent experiments.

\section{Figure 4. Heterogeneous MOMP induced more survival of cells after caspase activation.}

Candidate genes from the screen for the BAX/OMI-positive phenotype (RMDN3, ATG12 and BNIP3L) were chosen and deleted by CRISPR in U2OS cells and subjected to the clonogenic survival assay. The percentages of surviving cells were plotted. $\mathrm{P}$ values above the bar graphs show the difference in survival between the WT U2OS cells and each KO line.

Figure 5. Minority MOMP is enhanced by deletion of mitophagy- or mitochondria dynamics-related genes.

(A) Mitophagy defective penta-KO cells and their parental WT HeLa cells. (B) OPA1 KO MEFs and their matched WT MEFs. (C) MFN DKO MEFs and their matched WT MEFs. (D) DRP1 KO MEFs and DRP1-positive WT MEFs used in the MFN set in C. $\mathrm{n}$ denotes the number of independent experiments that were averaged. Error bars represent standard deviation. 

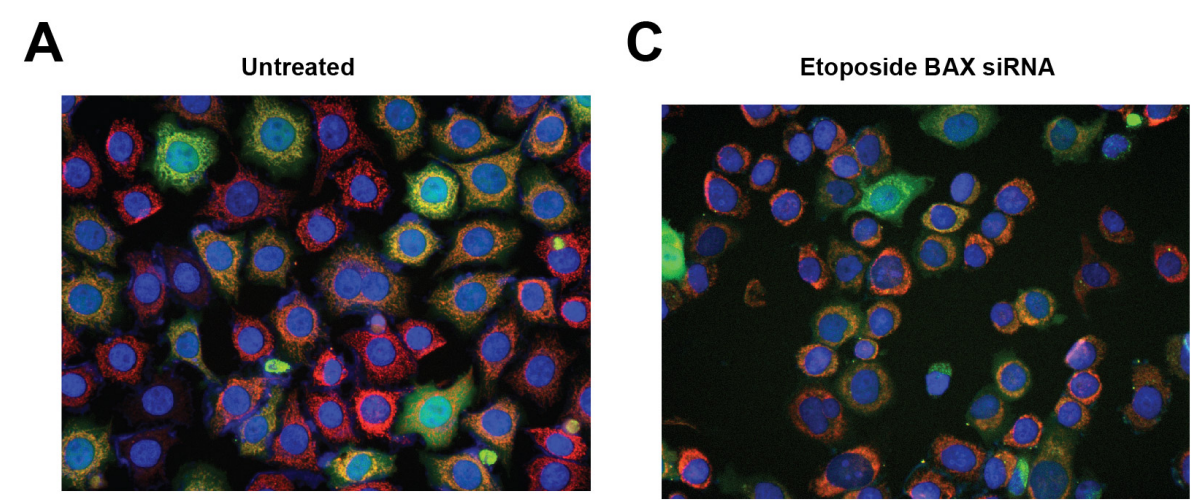

D

Etoposide OMA siRNA

Etoposide

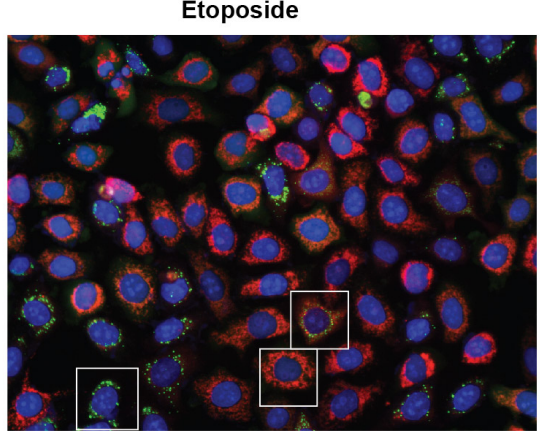

E
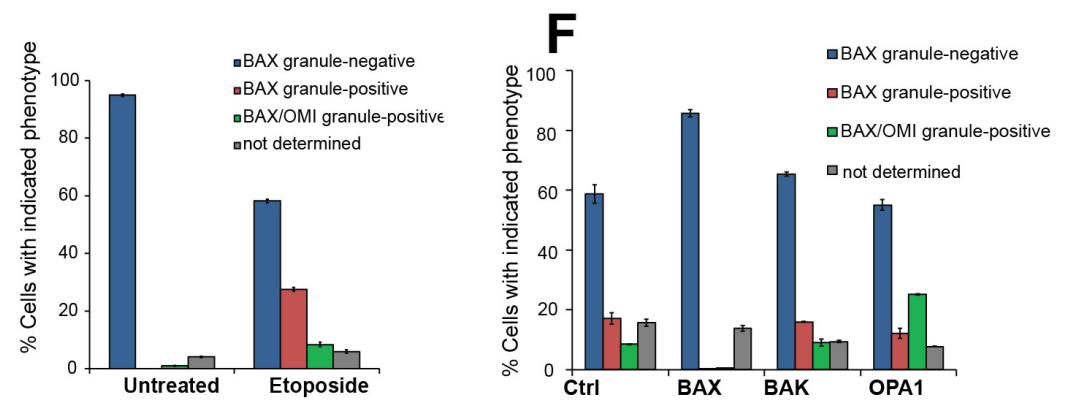

BAX granule- BAX granule- BAX/OMI granule-

positive

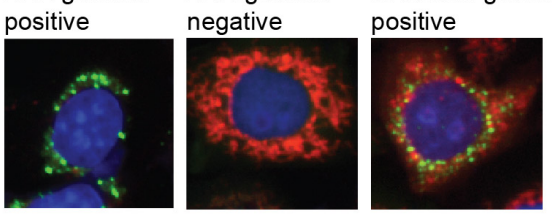

B

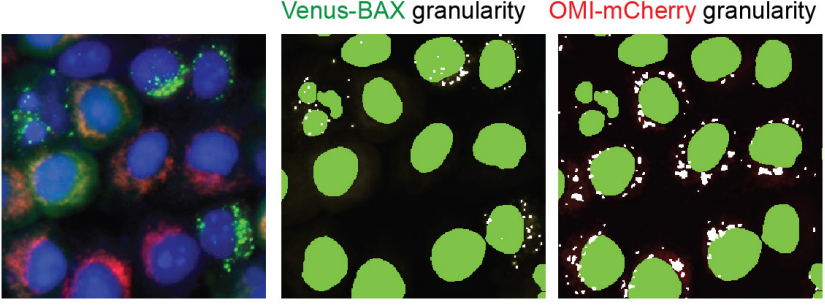

G

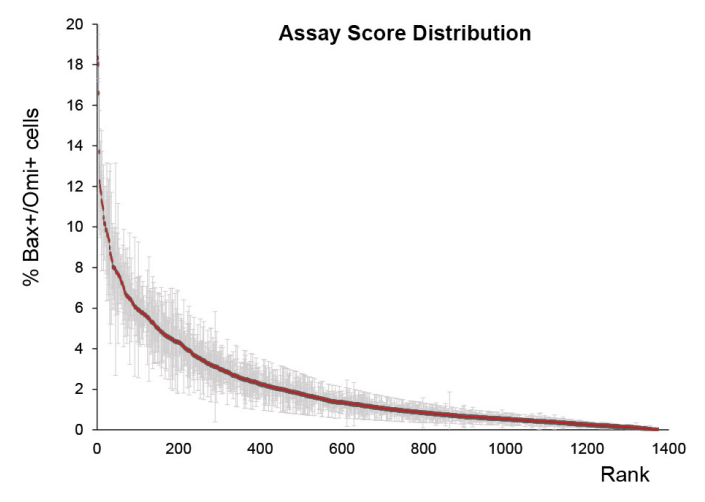

Figure 1 


\section{ABT treatment, $5 \mathrm{~h}$}

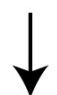

+ CellEvent caspase dye, $30 \mathrm{~min}$

Harvest and sort cells in the green gate into 48-well plates

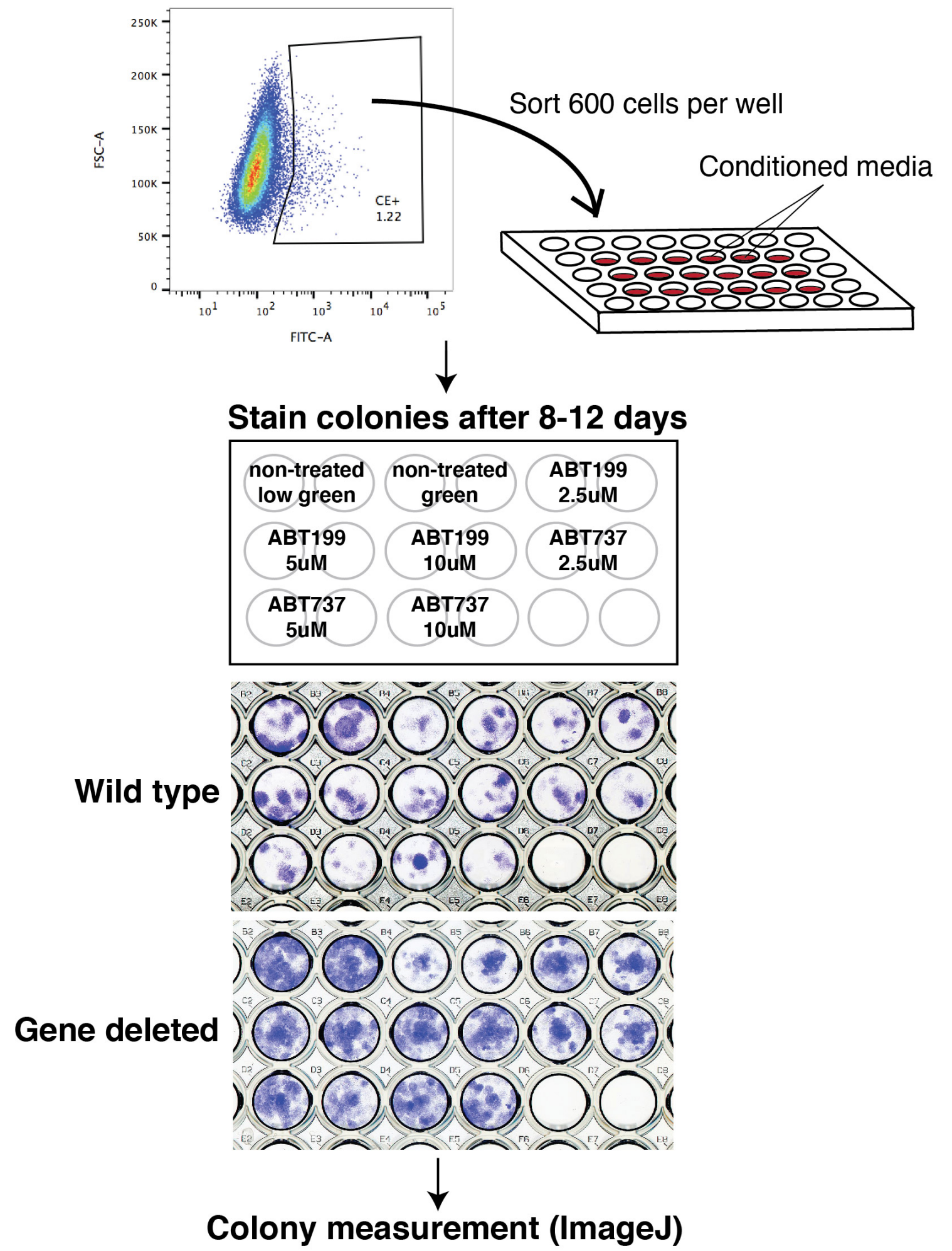

Normalized to the $100 \%$ growth (low green population of the non-treated sample) 


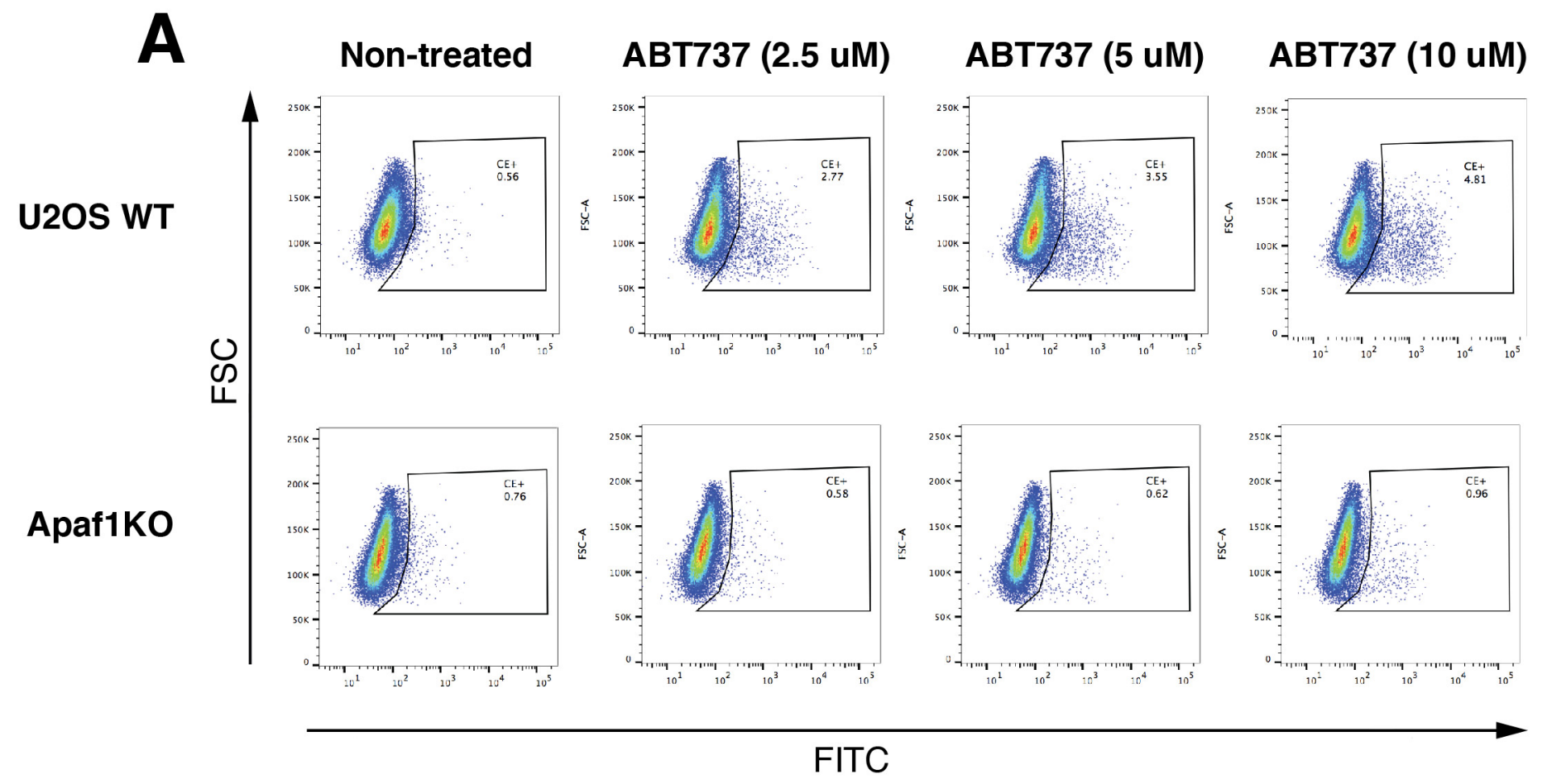

B

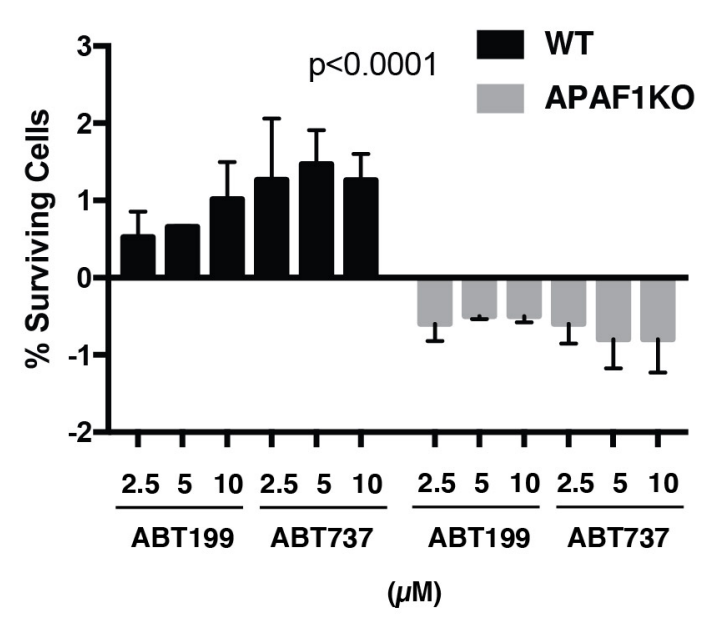

Figure 3 


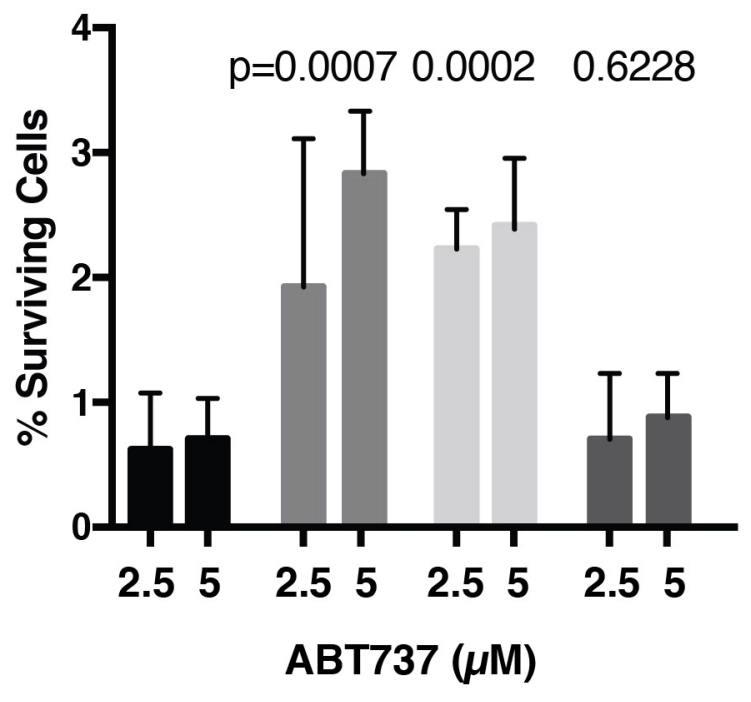

WT $(n=4)$

RMDN3 KO $(n=3)$ ATG12 KO $(n=2)$

BNIP3L KO $(n=2)$

Figure 4 
A

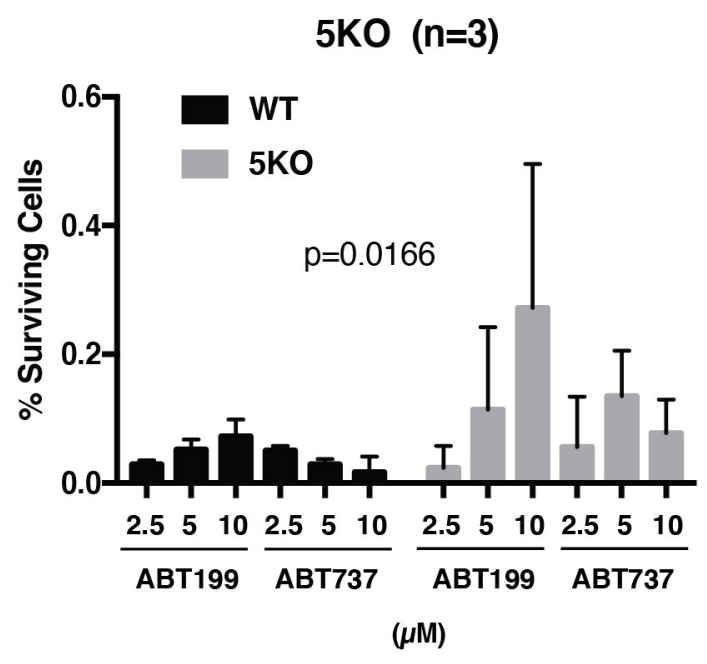

C

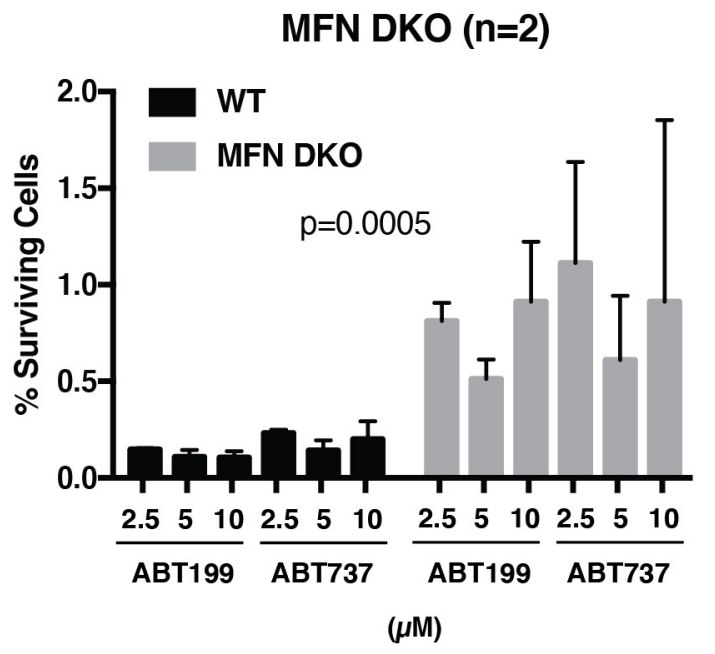

B OPA1 KO $(n=3)$

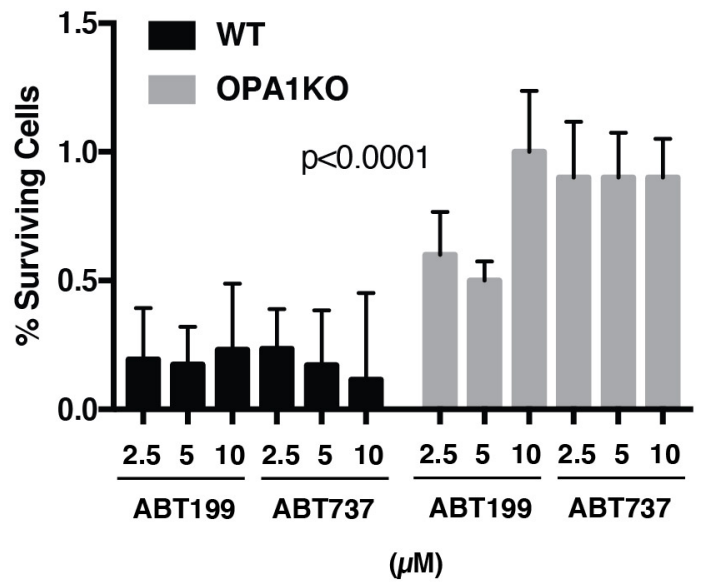

D DRP1 KO $(n=2)$

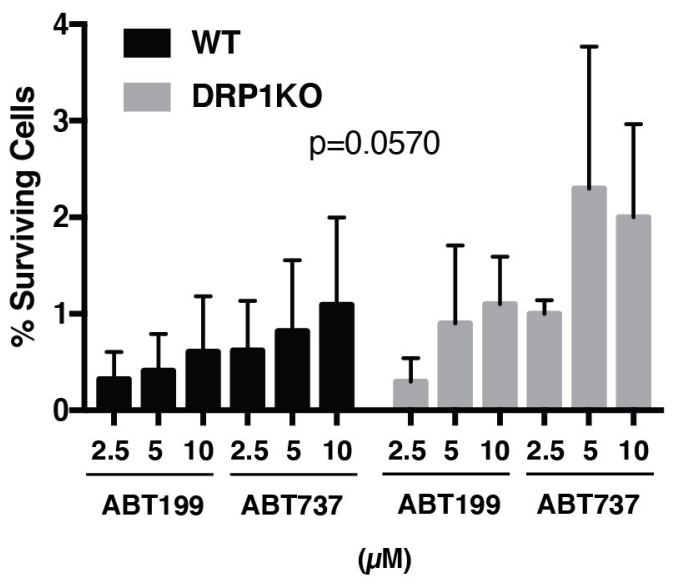

Figure 5 